\title{
Methyl Mercapturate Synthesis: An Efficient, Convenient and Simple Method
}

\section{Benoît Cossec *, Frédéric Cosnier and Manuella Burgart}

Institut National de Recherche et de Sécurité, Département Polluants et Santé, Rue du Morvan CS 60027, Vandoeuvre, 54519 cedex, France

* Author to whom correspondence should be addressed. E-mail: benoit.cossec@inrs.fr.

Received: 25 July 2008; in revised form: 9 September 2008 / Accepted: 26 September 2008 /

Published: 1 October 2008

\begin{abstract}
A safe and simple method for methyl $S$-arylmercapturate synthesis is described. Thirteen such compounds, to be used afterwards in metabolism studies, have been obtained with yields ranging from 71 to $99.6 \%$. These compounds were obtained using a sulfaMichael addition and synthesized by adding the corresponding thiophenols to a mixture composed of methyl 2-acetamidoacrylate (MAA), potassium carbonate and a phase transfer catalyst, Aliquat 336. MAA, the initial synthon, was itself isolated in quasi quantitative yield following a fully described synthesis.
\end{abstract}

Keywords: Mercapturic acids, Sulfa-Michael addition, Phase Transfer Catalysis, Biomarker

\section{Introduction}

Aromatic hydrocarbons belong to a broad family of chemical compounds which are widely used in many industrial applications, alone or in mixtures, and as solvents and starting products for chemical synthesis [1]. Along with the ever increasing awareness and knowledge about the toxicity of aromatic hydrocarbons [2-4], there is a growing need for assessing reliable and specific biomarkers and for the development of sensitive analytical methods dedicated to occupational exposure biomonitoring [5-10].

Mercapturic acids (MA), which are $N$-acetyl-L-cysteine- $S$-conjugates (or 2-acetamido-3-sulfanylpropionic acids according to IUPAC Nomenclature) are end products of the glutathione detoxification 
pathway (GSH). Since Van Doorn's preliminary studies [11], increasing attention has been paid to these minor metabolites. Over the last decades, several MA derived from aromatic hydrocarbons have been identified and their corresponding metabolic pathways have been explained [9-13]. In this respect, Angerer et al. $[13,14]$ demonstrated that toluene and xylenes are metabolised into MA using two different oxidation pathways (Scheme 1). As an example, toluene has been shown to interact with GSH by conjugation with reactive electrophilic intermediates. The nature of these intermediates depends on the first oxidation step catalysed by cytochrome P-450 enzymes and which may occur either at the side chain or at the aromatic ring. Side chain oxidation produces benzylic alcohol, which is enzymatically transformed into a sulphate ester [11], then to a $S$-benzylmercapturic acid (A) once conjugated with GSH. On the other hand, aromatic ring oxidation forms an arene oxide which leads to $S$-p-tolylmercapturic acid (B) after conjugation with GSH [13].

\section{Scheme 1.}

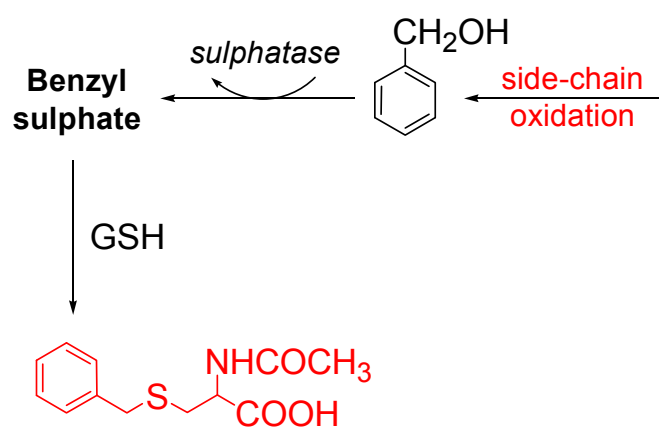

(A)

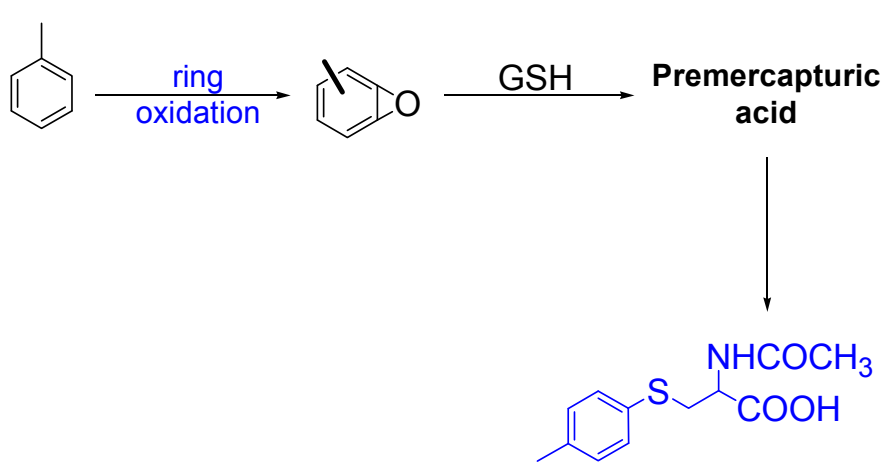

(B)

Several research teams have concluded that MAs are more selective and sensitive than traditional biomarkers, which are generally the major metabolites $[11,15,16]$. During the same period, the American Conference of Govermental Industrial Hygienists (ACGIH) and the Deutsche Forschungs Gemeinschaft (DFG) decided to publish a biological exposure index for $S$-phenylmercapturic acid as a biomarker of exposure to benzene [17, 18].

For the aforementioned reasons, a growing interest in the glutathione pathway and MA synthesis has emerged. While MAs coming from side chain oxidation are well described in the literature [1921], MAs resulting from ring oxidation are still being investigated in order to improve their synthesis. Scheme 2 reports the five main procedures for $S$-arylmercapturic acids (AMA) (or ester derivative) synthesis: the reaction of aryl diazonium salts with cysteine cuprous mercaptides or with $N$-acetyl-Lcysteine $[13,22,23]$, the copper-assisted or palladium-assisted nucleophilic substitution of aryliodides $[24,25]$, the nucleophilic substitution of aryl nitrate or nitroarylhalide [26-28], a Mitsunobu-type coupling reaction [29], and finally the process of Behringer et al. [30, 31]

Most of the processes already described gave low yields of complicated mixtures [13, 22, 23], or needed highly activated aromatics [28, 29, 31]. Moreover, they generally suffer from different drawbacks (longer reaction time, high temperature reaction, high starting material purity, rigorous dioxygen exclusion, tedious work-up) [30, 31]. 


\section{Scheme 2.}

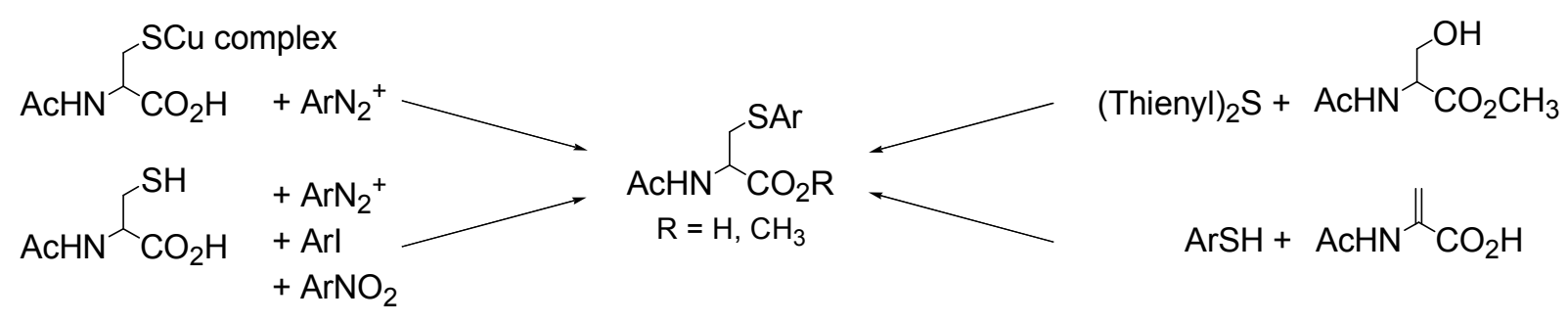

The aim of the present study was to provide gas chromatographic standards having a methyl $S$-arylmercapturate structure (AME) in order to use them in biological monitoring and metabolism studies. Thirteen compounds have thus been obtained in high yields from the procedure described in Scheme 3. This process is a two-step reaction starting from 2-acetamidoacrylic acid (product 1) and involving a smooth esterification of $\mathbf{1}$ followed by a sulfa-Michael addition [32] of the adequate thiophenol on the produced acrylate (product 2, MAA) in the presence of a phase-transfer catalyst [33].

Scheme 3.

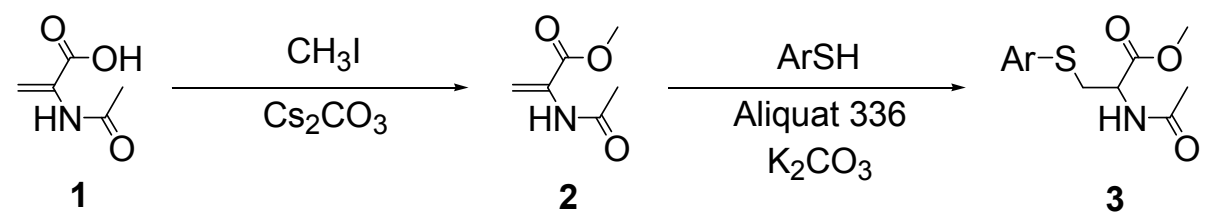

\section{Results and Discussion}

Methyl 2-acetamidoacrylate synthesis (MAA; 2)

Few procedures have been described in the past for MAA synthesis, and conventional acidic esterification attempts to obtain this ester often failed [34, 35]. This was probably due to the poor stability of the unsaturated structure. Rothstein et al. [34] first obtained MAA using the nucleophilic action of lead or sodium salts of compound 1 on iodomethane or dimethylsulphate, but yields never exceeded 52\%. Later on, Bueno et al. [36], using the potassium salt of 1, produced MAA in yields of up to $80 \%$, but in crude form. Another conventional reagent, diazomethane, is known to be inadequate for unsaturated carboxylic acid esterification, yielding quantitatively pyrazolines structures [37]. Other procedures reported in the literature involve methyl alaninate, cysteinate or serinate in more complicated retrosynthetic mechanisms [38-40].

As far as we are concerned, numerous methylation and esterification methods were investigated in the laboratory [41, 42], but only one reagent, cesium carbonate, was applied with success. Using experimental conditions described in the literature [43], the yield was gradually improved by increasing the ratio of iodomethane to compound 1 (Table 1). Finally, MAA was obtained quantitatively by carefully controlling the nucleophilic substitution and the time reaction. 
Table 1. MAA (2) synthesis.

\begin{tabular}{ccccc}
\hline Entry & $\begin{array}{c}\mathrm{Cs}_{2} \mathbf{C O}_{3} \\
\text { (equiv) }\end{array}$ & $\begin{array}{c}\mathbf{C H}_{3} \mathbf{I} \\
\text { (equiv } \\
\text { ) }\end{array}$ & Time (h) & Yield of 2 \\
& a (\%) \\
\hline 1 & 0.5 & 1.2 & 15 & 50 \\
2 & 0.5 & 2.0 & 15 & 65 \\
3 & 0.5 & 2.5 & 15 & 76 \\
4 & 0.5 & 2.5 & 3 & 99.7 \\
\hline \multicolumn{5}{c}{ a isolated yield }
\end{tabular}

Methyl S-p-toluylmercapturate (3a) synthesis: optimisation

Toluene was the first aromatic hydrocarbon to be examined when our biomonitoring studies began. Optimisation (Scheme 2, step 2) was therefore applied to obtain 3a, a mercapturate stemming from toluene ring oxidation (Scheme 1) [13]. The results concerning this particular optimisation are summarized in Table 2. Tetrabutylammonium hydrogensulfate (TBAHS), which generally performs successfully in all kinds of phase transfer catalysis (PTC) reactions [44, 45], was initially chosen as a catalyst. Using liquid-liquid conditions (entry 1), a complete deesterification of 2 unfortunately resulted. This negative result prompted us on the one hand to suppress water and to use a solid-liquid process [46-48] and on another hand to replace $\mathrm{NaOH}$ by $\mathrm{KOH}$. Three reaction-condition parameters, i.e. the organic phase, the basic anion and the catalyst used, were then successively modified to improve the yields obtained.

Three solvents were thus investigated and put through the procedure. From the results of runs 3,4 and 5 , toluene may be considered as the reference solvent, other solvents appearing less suitable. This result is not in accordance with Herriott et al. who suggested that the reaction rate in PTC should be directly related to solvent polarity [49]. When the results of runs 9 and 10 were analysed, the relationship between solvent and yield did not appear so simple. The results observed with THF (tetrahydrofuran), which is a polar solvent, were similar to those obtained with toluene.

The investigations then moved on to screening two different bases [50]. The results obtained in runs 2 and 3, and comparatively in runs 8 and 9, clearly demonstrate that $\mathrm{K}_{2} \mathrm{CO}_{3}$ is superior to $\mathrm{KOH}$ in terms of compound 3a formation. Furthermore, the reactions with this latter base were by far the cleanest, producing only a very minor amounts of by-products.

Two other PTC catalysts, cetyltrimethyl ammonium bromide (CTAB) and methyltrioctyl ammonium chloride (Aliquat 336), were then successively tested to conduct the sulfa-Michael addition (entries 3, 7, 8 and 9). Aliquat 336 emerged as the most active catalyst for our process (lowest quantities, highest rate).

Finally, the reaction was performed with a excess of reagent (entry 11). In fact, it is well known that arylmercaptans are particularly sensitive to oxidation. With an excess of $p$-cresol a quasi complete conversion was observed. To confirm the catalytic effect, the reaction was then carried out using the conditions described in entry 11 and avoiding the use of the selected PTC catalyst. In contrast with the preceding results, no reaction at all was observed at room temperature over a 24-hour stirring period. 
In summary, appropriate solid-liquid phase-transfer catalysis conditions have been determined to carry out the addition of p-thiophenol to MAA, THF and toluene emerging as the solvents of choice. The addition takes place rapidly by successively adding a catalytic quantity of the basic anion $\left(\mathrm{K}_{2} \mathrm{CO}_{3}\right)$ and then the catalyst (Aliquat 336) to the reaction mixture, which yields $\mathbf{3 a}$, the desired product, quasi quantitatively.

Table 2. Optimisation of the reaction conditions for the addition of $p$-thiocresol to compound 2.

\begin{tabular}{|c|c|c|c|c|c|c|}
\hline Entry & $\begin{array}{c}p \text {-Thiocresol } \\
(\mathrm{mmol})\end{array}$ & $\begin{array}{c}\text { Base } \\
(\mathrm{mmol})\end{array}$ & $\begin{array}{c}\text { PTC } \\
(\mathbf{m m o l})\end{array}$ & Solvent & $\begin{array}{c}\text { Time } \\
\text { (h) }\end{array}$ & $\begin{array}{c}\text { \%Yield } \\
\text { for 3a }\end{array}$ \\
\hline 1 & 1 & $\mathrm{NaOH} 1 \mathrm{M} / \mathrm{H}_{2} \mathrm{O}$ & TBAHS (0.2) & $\mathrm{CH}_{2} \mathrm{Cl}_{2} / \mathrm{H}_{2} \mathrm{O}$ & 48 & $100 \%$ of 2 \\
\hline 2 & 1 & $\mathrm{NaOH}(1.4)$ & TBAHS (0.2) & THF & 48 & 1 \\
\hline 3 & 1 & $\mathrm{KOH}(1.2)$ & TBAHS (0.2) & THF & 18 & 22 \\
\hline 4 & 1 & $\mathrm{KOH}(1.4)$ & TBAHS (0.2) & Acetonitrile & 18 & 22 \\
\hline 5 & 1 & $\mathrm{KOH}(1.2)$ & TBAHS (0.2) & Toluene & 18 & 33 \\
\hline 6 & 1 & $\mathrm{KOH}(1.2)$ & CTAB $(0.2)$ & Toluene & 48 & 31 \\
\hline 7 & 1 & $\mathrm{KOH}(1.2)$ & CTAB $(0.2)$ & THF & 18 & 49 \\
\hline 8 & 1 & $\mathrm{KOH}(1.2)$ & Aliquat $336(0.08)$ & THF & 18 & 6 \\
\hline 9 & 1 & $\mathrm{~K}_{2} \mathrm{CO}_{3}(0.3)$ & Aliquat $336(0.08)$ & THF & 18 & 63 \\
\hline 10 & 1 & $\mathrm{~K}_{2} \mathrm{CO}_{3}(0.3)$ & Aliquat $336(0.08)$ & Toluene & 18 & 61 \\
\hline 11 & 1.4 & $\mathrm{~K}_{2} \mathrm{CO}_{3}(0.3)$ & Aliquat $336(0.08)$ & Toluene & 5 & 99.6 \\
\hline
\end{tabular}

Amount of 2 used: $1 \mathrm{mmol}$

Application to the synthesis of various AMEs (3b-m)

Using the optimised conditions established for 3a, the Michael addition was applied to numerous thiophenols (Table 3). As expected from previous results, thiophenols bearing para but also ortho electron donating groups reacted quickly, giving the corresponding AMEs 3b-d in remarkable yields in less than 5 hours. Similarly, thiophenols bearing two electron donating groups afforded AMEs 3e-h in satisfactory yields, but needed generally slightly longer reaction times.

In order to complete the knowledge of this procedure, several other thiophenols were then investigated. It was successively demonstrated that a donating group was not required (3i), and, that despite the electron withdrawing group presence, $\mathbf{3} \mathbf{j}-\mathbf{l}$ can be obtained in relatively satisfactory yields with reaction times ranging from 5 to 18 hours. In addition, and unexpectedly, a thiophenol bearing five electron withdrawing groups $(\mathbf{3 m})$ gave similar results.

Finally, our procedure is safe, simple, convenient, and compatible with numerous substrates and, by and large, produced AMEs in higher yields compared to other protocols [22-31]. Furthermore, availability and low cost of Aliquat 336 are an additional favourable factor to consider. 
Table 3. Methyl S-arylmercapturates (AME) synthesis.

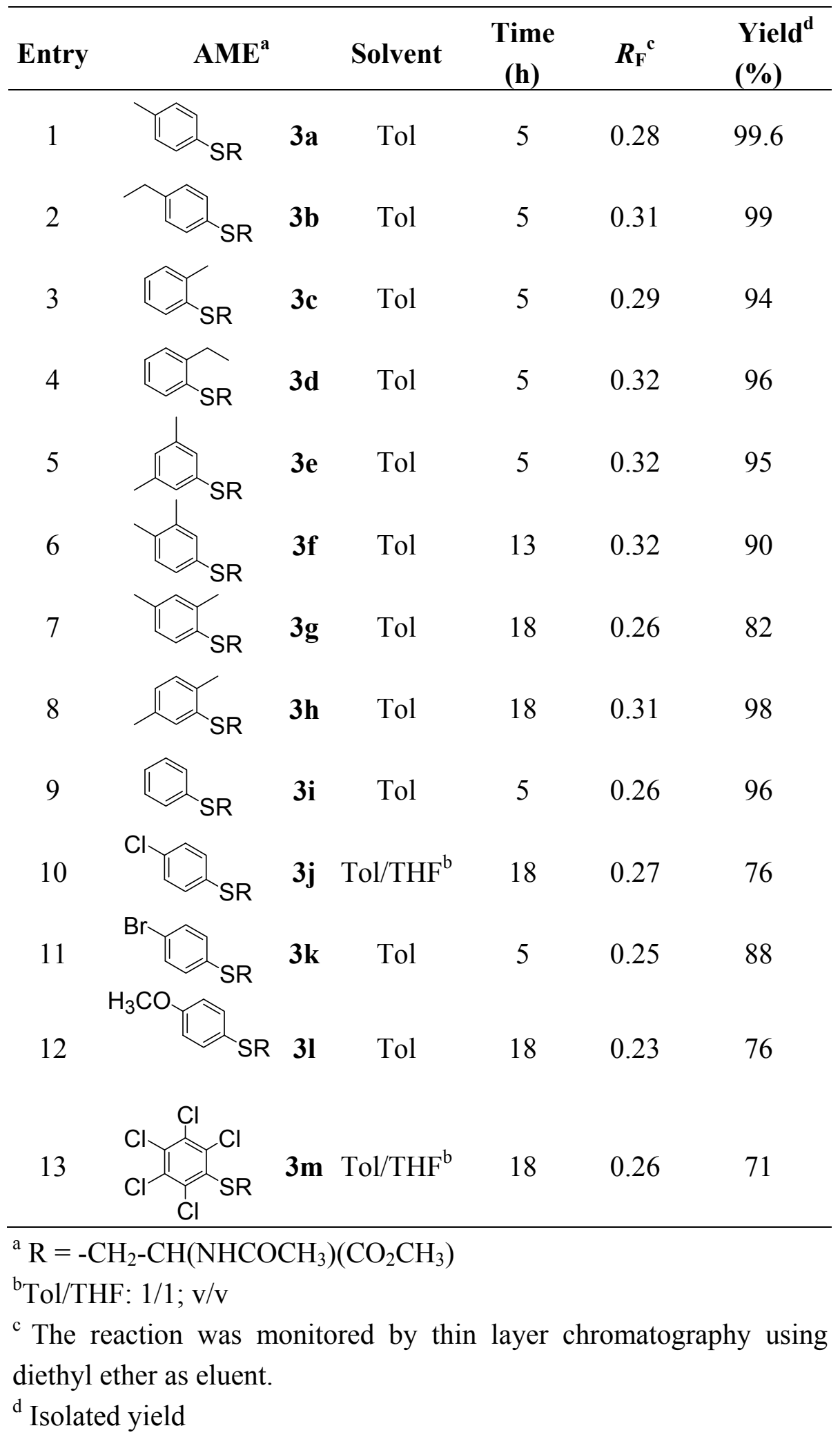

\section{Conclusions}

Methyl $S$-arylmercapturates have been synthesized according to a two-step reaction starting from compound 1 (Scheme 2) and involving a sulfa-Michael addition whose reactivity was enhanced by 
adding a phase transfer catalyst. A simple and efficient synthesis of MAA (2), the intermediary synthon, is described. The complete method proceeds smoothly with typical yields ranging from 71 to $99.6 \%$, and may be applied to various thiophenols bearing electron donating groups or electron withdrawing groups. These compounds were then used as standards in gas chromatographic analysis and in metabolism studies.

\section{Experimental}

\section{General}

2-Acetamidoacrylic acid (1), thiophenol, chlorothiophenol, pentachlorothiophenol, 4-toluenethiol, 2-ethylthiophenol, 2-toluenethiol, cesium carbonate, tetrabutylammonium hydrogensulfate (TBAHS), cetyltrimethyl ammonium bromide (CTAB) and methyltrioctyl ammonium chloride (Aliquat 336) were purchased from Sigma-Aldrich-Fluka. 4-bromothiophenol, 2,4-dimethylthiophenol, 2,5dimethyl-thiophenol, 3,4-dimethylthiophenol and 3,5-dimethylthiophenol were all available from Acros Organics (Fisher Bioblock, France). Finally, 4-ethylthiophenol was obtained from Avocado (Interchim, France). All the other chemicals were of analytical grade. ${ }^{1} \mathrm{H}-\mathrm{NMR}$ and ${ }^{13} \mathrm{C}-\mathrm{NMR}$ spectra were obtained on a Bruker AM 400 spectrometer operated at 400 and $100 \mathrm{MHz}$, respectively. The chemical shifts are reported in parts per million relative to TMS. The melting points (m.p.) were determined with an Electrothermal digital melting point apparatus and were uncorrected. The elemental analyses (EA) were performed by the Central Analysis Department of CNRS (Vernaison, France). The mass spectra (MS) were collected in electronic impact mode $(70 \mathrm{eV})$ on a Varian 1200 MS/MS mass spectrometer coupled to a Varian 3800 gas chromatograph (GC). A Varian DB-5MS column ( $30 \mathrm{~m} ; 0.25 \mathrm{~mm} ; 0.25 \mu \mathrm{m})$ was used for the AME chromatography. The carrier gas was helium $\left(1 \mathrm{~mL} / \mathrm{min}\right.$ ). The injector (split ratio $=30$ ) was held at $250{ }^{\circ} \mathrm{C}$. The $\mathrm{GC}$ oven program was: $70{ }^{\circ} \mathrm{C}$ initial temperature, isothermal for $1.5 \mathrm{~min}$, then $15^{\circ} \mathrm{C} / \mathrm{min}$ to $250{ }^{\circ} \mathrm{C}$, and finally isothermal for $7 \mathrm{~min}$. Thin-layer chromatography (TLC) was carried out on Merck 60 F254 precoated silica gel plates $(0.25 \mathrm{~mm})$ using diethyl ether $(100 \%)$ as eluent $\left(R_{\mathrm{F}}\right.$ in Table 3$)$. Spots were visualized successively with a UV lamp $(\lambda=254 \mathrm{~nm})$ and iodine vapors.

\section{Methyl 2-acetamidoacrylate synthesis (MAA; 2)}

$\mathrm{Cs}_{2} \mathrm{CO}_{3}(4.2 \mathrm{mmol})$ was added to $1(1368 \mathrm{mg}, 8.4 \mathrm{mmol})$ dissolved in $\mathrm{MeOH}(20 \mathrm{~mL})$, and the mixture was stirred at room temperature for one hour. The methanolic solution was concentrated until dryness. Iodomethane (42 mmol; $2.7 \mathrm{~mL}$ ), in controlled portions and undergoing constant stirring, was added over a two-hour period to the residue dissolved in DMF $(30 \mathrm{~mL})$. The reaction mixture was finally concentrated to dryness, and the crude residue, purified over a silica gel column using diethyl ether $\left(\mathrm{Et}_{2} \mathrm{O} 100 \%, R_{\mathrm{F}}=0.55\right)$ as eluent, gave $1199 \mathrm{mg}$ of 2 as white needles, i.e. a yield of $99.7 \%$

Methyl 2-acetamidoacrylate (2). White solid, mp: 52-53 ${ }^{\circ} \mathrm{C} ;{ }^{1} \mathrm{H}-\mathrm{NMR}\left(\mathrm{CDCl}_{3}\right): \delta(\mathrm{ppm}) 2.11(\mathrm{~s}, 3 \mathrm{H}$, $\left.\mathrm{COCH}_{3}\right), 3.82\left(\mathrm{~s}, 3 \mathrm{H}, \mathrm{OCH}_{3}\right), 5.85\left(\mathrm{~s}, 1 \mathrm{H}, \mathrm{CH}_{2}\right), 6.57\left(\mathrm{~s}, 1 \mathrm{H}, \mathrm{CH}_{2}\right), 7.72(\mathrm{~s}, 1 \mathrm{H}, \mathrm{NH}) ;{ }^{13} \mathrm{C}-\mathrm{NMR}$ $\left(\mathrm{CDCl}_{3}\right): \delta(\mathrm{ppm}) 24.56\left(\mathrm{CH}_{3}\right), 52.88\left(\mathrm{CH}_{3}\right), 108.63\left(\mathrm{CH}_{2}\right), 130.85\left(\mathrm{C}=\mathrm{CH}_{2}\right), 164.56\left(\mathrm{CO}_{2}\right), 168.74$ 
(CO); IR: $v\left(\mathrm{~cm}^{-1}\right)$ 3364, 3155, 3057, 2963, 1813, 1708, 1678, 1629, 1512, 1428, 1369, 1327, 1246, 1208, 1172, 1038, 995, 977, 905, 856, 809; MS (m/z, EI): 143 (M+ 66), 111 (44), 101 (100), 71 (27), 43 (88), 42 (63); Anal. Calcd for $\mathrm{C}_{6} \mathrm{H}_{9} \mathrm{NO}_{3}$ : C, 50.35; H, 6.34; N, 9.79; O, 33,53; Found: C, 50.41; H, $6.37 ; \mathrm{N}, 9.39 ; \mathrm{O}, 33,30$.

\section{General procedure for methyl S-arylmercapturate (AME) synthesis: compounds 3a-m}

Compounds $\mathbf{3 b}$ to $\mathbf{3} \mathbf{m}$ were synthesized using the parameters defined for $\mathbf{3 a}$ in Table 2 entry 11 and in Table 3. Briefly, adequate thiophenol $(1.4 \mathrm{mmol})$, Aliquat 336 (32 $\mathrm{mg}, 0.08 \mathrm{mmol})$ and finally powdered potassium carbonate $(41 \mathrm{mg}, 0.3 \mathrm{mmol})$ were added respectively to 2 (143 $\mathrm{mg}, 1 \mathrm{mmol})$ dissolved in toluene $(30 \mathrm{~mL}$, except if otherwise indicated in Table 3$)$. The reaction mixture was stirred at room temperature and regularly monitored by thin layer chromatography using $\mathrm{Et}_{2} \mathrm{O}$ as eluent $\left(\mathrm{R}_{\mathrm{F}}\right.$, Table 3$)$. The mixture filtered, the crude product, isolated after solvent removal, was subjected to silica gel column chromatography using $\mathrm{Et}_{2} \mathrm{O}$ as eluent. Yields are given in Table 3.

2-Acetamido-3-p-tolylsulfanylpropionic acid methyl ester (3a). White solid, mp: $84-85^{\circ} \mathrm{C}$; ${ }^{1} \mathrm{H}-\mathrm{NMR}$ $\left(\mathrm{CD}_{3} \mathrm{OD}\right): \delta(\mathrm{ppm}) 1.88\left(\mathrm{~s}, 3 \mathrm{H}, \mathrm{COCH}_{3}\right), 2.31$ (s, 3H, $\left.\mathrm{CH}_{3}-\mathrm{Ar}\right), 3.28-3.44$ (m, 2H, $\left.\mathrm{SCH}_{2}\right), 3.56(\mathrm{~s}, 3 \mathrm{H}$, $\left.\mathrm{O}-\mathrm{CH}_{3}\right), 4.60-4.63\left(\mathrm{~m}, 1 \mathrm{H}, \mathrm{CH}-\mathrm{CH}_{2}\right), 4.81-4.85(\mathrm{~m}, 1 \mathrm{H}), 7.09-7.32(\mathrm{~m}, 4 \mathrm{H}, \mathrm{ArH})$; ${ }^{13} \mathrm{C}-\mathrm{NMR}$ $\left(\mathrm{CD}_{3} \mathrm{OD}\right): \delta(\mathrm{ppm}) 20.1\left(\mathrm{CH}_{3}\right), 21.4\left(\mathrm{CH}_{3}\right), 36.2\left(\mathrm{CH}_{2}\right), 51.8\left(\mathrm{CH}_{3}\right), 52.7(\mathrm{CH}), 129.9\left(\mathrm{CH}_{\mathrm{Ar}}\right), 131.4$ $\left(\mathrm{CH}_{\mathrm{Ar}}\right), 131.6(\mathrm{C}), 137.5(\mathrm{C}), 171.4(\mathrm{CO}), 172.2(\mathrm{CO})$; IR: $v\left(\mathrm{~cm}^{-1}\right)$ 3322, 3075, 2984, 2944, 2931, 2843, 1727, 1645, 1543, 1495, 1423, 1344, 1321, 1248, 1179, 1089, 1036, 803; MS (m/z, EI): 267 $\left(\mathrm{M}^{+}, 15\right), 208$ (80), 149 (30), 43 (100); Anal. Calcd for $\mathrm{C}_{12} \mathrm{H}_{17} \mathrm{NO}_{3} \mathrm{~S}$; C 58.40, H 6.41, N 5.24, O 17.95, S 11.99; Found: C 58.66, H 6.48, N 5.14, O 18.40, S 12.16.

2-Acetamido-3-(4-ethylphenylsulfanyl)-propionic acid methyl ester (3b). White solid, $\mathrm{mp}$ : $64-65^{\circ} \mathrm{C}$; ${ }^{1} \mathrm{H}-\mathrm{NMR}\left(\mathrm{CD}_{3} \mathrm{OD}\right): \delta(\mathrm{ppm}) 1.19\left(\mathrm{t},{ }^{3} J=7.6 \mathrm{~Hz}, 3 \mathrm{H}, \mathrm{CH}_{3}\right), 1.92\left(\mathrm{~s}, 3 \mathrm{H}, \mathrm{COCH}_{3}\right), 2.59\left(\mathrm{q},{ }^{3} J=7.6\right.$ $\left.\mathrm{Hz}, 2 \mathrm{H}, \mathrm{CH}_{2}-\mathrm{CH}_{3}\right), 3.18\left(\mathrm{dd},{ }^{2} J=14 \mathrm{~Hz},{ }^{3} J=7.8 \mathrm{~Hz}, 1 \mathrm{H}, \mathrm{SCH}_{2}\right), 3.34\left(\mathrm{dd},{ }^{2} J=14 \mathrm{~Hz},{ }^{3} J=4.8 \mathrm{~Hz}, 1\right.$ $\left.\mathrm{H}, \mathrm{SCH}_{2}\right), 3.60\left(\mathrm{~s}, 3 \mathrm{H}, \mathrm{OCH}_{3}\right), 4.55\left(\mathrm{dd},{ }^{3} J=7.8 \mathrm{~Hz},{ }^{3} J=4.8 \mathrm{~Hz}, 1 \mathrm{H}, \mathrm{CH}_{2}-\mathrm{CH}\right), 4.84(\mathrm{~s}, 1 \mathrm{H}, \mathrm{NH})$, 7.13-7.16 (m, 2 H, 2-Ar-H), 7.32-7.35 (m,2 H, 2 Ar-H); ${ }^{13} \mathrm{C}-\mathrm{NMR}\left(\mathrm{CD}_{3} \mathrm{OD}\right): \delta(\mathrm{ppm}) 16.2\left(\mathrm{CH}_{3}\right), 22.4$ $\left(\mathrm{CH}_{3}\right), 29.5\left(\mathrm{CH}_{2}\right), 37.3\left(\mathrm{CH}_{2}\right), 53.0\left(\mathrm{CH}_{3}\right), 53.8(\mathrm{CH}), 129.8\left(\mathrm{CH}_{\mathrm{Ar}}\right), 131.0\left(\mathrm{C}_{\mathrm{Ar}}\right), 132.7\left(\mathrm{CH}_{\mathrm{Ar}}\right), 145.0$ $\left(\mathrm{C}_{\mathrm{Ar}}\right), 172.4(\mathrm{CO}), 173.2(\mathrm{CO})$; IR: $v\left(\mathrm{~cm}^{-1}\right)$ 3318, 3076, 2969, 2946, 2934, 1723, 1637, 1546, 1496, 1438, 1422, 1347, 1321, 1248, 1219, 1180, 1091, 1036, 1017, 814, 696; MS (m/z, EI): $281\left(\mathrm{M}^{+}, 26\right)$, 222 (100), 207 (10), 190 (6), 163 (53), 151 (55), 136 (78), 123 (29), 105 (25), 88 (52), 77 (60), 59 (51); Anal. Calcd for $\mathrm{C}_{14} \mathrm{H}_{19} \mathrm{NO}_{3} \mathrm{~S}$; C 59.76, H 6.81, N 4.98, O 17.06, S 11.40; Found: C 59.82, H 6.79, N 4.90, O 17.35, S 11.46.

2-Acetamido-3-o-tolylsulfanylpropionic acid methyl ester (3c). White solid, mp: $66-67^{\circ} \mathrm{C}$; ${ }^{1} \mathrm{H}-\mathrm{NMR}$ $\left(\mathrm{CD}_{3} \mathrm{OD}\right): \delta(\mathrm{ppm}) 1.95$ (s, $\left.3 \mathrm{H}, \mathrm{COCH}_{3}\right), 2.38\left(\mathrm{~s}, 3 \mathrm{H}, \mathrm{CH}_{3}-\mathrm{Ar}\right), 3.21\left(\mathrm{dd},{ }^{2} J=14.0 \mathrm{~Hz},{ }^{3} J=8.0 \mathrm{~Hz}, 1\right.$ $\left.\mathrm{H}, \mathrm{SCH}_{2}\right), 3.39\left(\mathrm{dd},{ }^{2} J=14.0 \mathrm{~Hz},{ }^{3} J=4.9 \mathrm{~Hz}, 1 \mathrm{H}, \mathrm{SCH}_{2}\right), 3.64\left(\mathrm{~s}, 3 \mathrm{H}, \mathrm{OCH}_{3}\right), 4.58\left(\mathrm{dd},{ }^{3} J=8.0 \mathrm{~Hz}\right.$, $\left.{ }^{3} J=4.9 \mathrm{~Hz}, 1 \mathrm{H}, \mathrm{CH}-\mathrm{CH}_{2}\right), 7.12-7.22(\mathrm{~m}, 3 \mathrm{H}, \mathrm{Ar}-\mathrm{H}), 7.41-7.43(\mathrm{~m}, 1 \mathrm{H}, \mathrm{Ar}-\mathrm{H}) ;{ }^{13} \mathrm{C}-\mathrm{NMR}$ $\left(\mathrm{CD}_{3} \mathrm{OD}\right): \delta(\mathrm{ppm}) 19.6\left(\mathrm{CH}_{3}\right), 21.2\left(\mathrm{CH}_{3}\right), 34.8\left(\mathrm{CH}_{2}\right), 52.0\left(\mathrm{CH}_{3}\right), 52.7(\mathrm{CH}), 126.9\left(\mathrm{CH}_{\mathrm{Ar}}\right), 127.4$ $\left(\mathrm{CH}_{\mathrm{Ar}}\right), 130.5\left(\mathrm{CH}_{\mathrm{Ar}}\right), 130.7\left(\mathrm{CH}_{\mathrm{Ar}}\right), 134.1\left(\mathrm{C}_{\mathrm{Ar}}\right), 139.1\left(\mathrm{C}_{\mathrm{Ar}}\right), 171.3(\mathrm{CO}), 172.2(\mathrm{CO}) ; \mathrm{IR}: v\left(\mathrm{~cm}^{-1}\right)$ 
3311, 3069, 2952, 2940, 2838, 1736, 1650, 1542, 1432, 1335, 1223, 1168, 1036, 744; MS (m/z, EI): 267 (M+13), 208 (100), 149 (56), 137 (44), 134 (61), 91 (18), 43 (26); Anal. Calcd for $\mathrm{C}_{12} \mathrm{H}_{17} \mathrm{NO}_{3} \mathrm{~S}$; C 58.40, H 6.41, N 5.24, O 17.95, S 11.99; Found: C 58.28, H 6.45, N 5.08, O 18.19, S 12.19.

2-Acetamido-3-(2-ethylphenylsulfanyl)propionic acid methyl ester (3d). White solid, $\mathrm{mp}: 55-56{ }^{\circ} \mathrm{C} ;{ }^{1} \mathrm{H}$ NMR ( $\left.\mathrm{CD}_{3} \mathrm{OD}\right): \delta(\mathrm{ppm}) 1,18\left(\mathrm{t},{ }^{3} J=7.5 \mathrm{~Hz}, 3 \mathrm{H}, \mathrm{CH}_{3}-\mathrm{CH}_{2}\right), 1.94\left(\mathrm{~s}, 3 \mathrm{H}, \mathrm{COCH}_{3}\right), 2.78\left(\mathrm{q},{ }^{3} J=7.5\right.$ $\left.\mathrm{Hz}, 2 \mathrm{H}, \mathrm{CH}_{2}-\mathrm{CH}_{3}\right), 3.19\left(\mathrm{dd},{ }^{2} J=13.9 \mathrm{~Hz},{ }^{3} J=8.1 \mathrm{~Hz}, 1 \mathrm{H}, \mathrm{SCH}_{2}\right), 3.39\left(\mathrm{dd},{ }^{2} J=13.9 \mathrm{~Hz},{ }^{3} J=5,0\right.$ $\left.\mathrm{Hz}, 1 \mathrm{H}, \mathrm{SCH}_{2}\right), 3.62$ (s, 3H, $\left.\mathrm{CH}_{3}-\mathrm{Ar}\right), 4.55\left(\mathrm{dd},{ }^{3} \mathrm{~J}=8.1 \mathrm{~Hz},{ }^{3} \mathrm{~J}=5.0 \mathrm{~Hz}, 1 \mathrm{H}, \mathrm{CH}-\mathrm{CH}_{2}\right), 7.15-7.21$ (m, $3 \mathrm{H}, \mathrm{Ar}-\mathrm{H}), 7.41-7.44$ (m, $1 \mathrm{H}, \mathrm{Ar}-\mathrm{H}) ;{ }^{13} \mathrm{C}-\mathrm{NMR}\left(\mathrm{CD}_{3} \mathrm{OD}\right): \delta(\mathrm{ppm}) 15.7\left(\mathrm{CH}_{3}\right), 22.4\left(\mathrm{CH}_{3}\right), 28.2$ $\left(\mathrm{CH}_{2}\right), 36.5\left(\mathrm{CH}_{2}\right), 53.0\left(\mathrm{CH}_{3}\right), 53.7(\mathrm{CH}), 127.8\left(\mathrm{CH}_{\mathrm{Ar}}\right), 128.4\left(\mathrm{CH}_{\mathrm{Ar}}\right), 130.1\left(\mathrm{CH}_{\mathrm{Ar}}\right), 131.9\left(\mathrm{CH}_{\mathrm{Ar}}\right)$, $134.6\left(\mathrm{C}_{\mathrm{Ar}}\right), 146.3\left(\mathrm{C}_{\mathrm{Ar}}\right), 172.5(\mathrm{CO}), 173.3(\mathrm{CO})$; IR: $v\left(\mathrm{~cm}^{-1}\right)$ 3280, 3058, 2964, 2872, 1747, 1655, 1541, 1437, 1373, 1214, 1128, 1027, 752; MS (m/z, EI): $281\left(\mathrm{M}^{+}, 42\right), 222$ (88), 151 (30), 148 (100), 144 (25), 136 (45), 91 (12), 43 (21); Anal. Calcd for $\mathrm{C}_{14} \mathrm{H}_{19} \mathrm{NO}_{3} \mathrm{~S}$; C 59.76, H 6.81, N 4.98, O 17.06, S 11.40; Found: C 59.39, H 6.76, N 4.88, O 17.46, S 11.74.

2-Acetamido-3-(3,5-dimethylphenylsulfanyl)propionic acid methyl ester (3e). White solid, mp: 73$74^{\circ} \mathrm{C}$; ${ }^{1} \mathrm{H}-\mathrm{NMR}\left(\mathrm{CD}_{3} \mathrm{OD}\right): \delta(\mathrm{ppm}) 1.92\left(\mathrm{~s}, 3 \mathrm{H}, \mathrm{COCH}_{3}\right), 2.25\left(\mathrm{~s}, 6 \mathrm{H}, 2 \mathrm{CH}_{3}-\mathrm{Ar}\right), 3.18$ (dd, ${ }^{2} J=14.2$ $\left.\mathrm{Hz},{ }^{3} J=7.6 \mathrm{~Hz}, 1 \mathrm{H}, \mathrm{SCH}_{2}\right), 3.35\left(\mathrm{dd},{ }^{2} J=14.2 \mathrm{~Hz},{ }^{3} J=4.8 \mathrm{~Hz}, 1 \mathrm{H}, \mathrm{SCH}_{2}\right), 3.62\left(\mathrm{~s}, 3 \mathrm{H}, \mathrm{OCH}_{3}\right)$, $4.57\left(\mathrm{dd},{ }^{3} \mathrm{~J}=7.6 \mathrm{~Hz},{ }^{3} \mathrm{~J}=4.8 \mathrm{~Hz}, 1 \mathrm{H}, \mathrm{CH}_{2}-\mathrm{CH}\right), 4.84(\mathrm{~s}, 1 \mathrm{H}, \mathrm{NH}), 6.85$ (m, $\left.1 \mathrm{H}, 1 \mathrm{Ar}-\mathrm{H}\right), 7.01$ (m, 2 $\mathrm{H}, 2 \mathrm{Ar}-\mathrm{H}) ;{ }^{13} \mathrm{C}-\mathrm{NMR}\left(\mathrm{CD}_{3} \mathrm{OD}\right): \delta(\mathrm{ppm}) 21.4\left(2 \times \mathrm{CH}_{3}\right), 22.4\left(\mathrm{CH}_{3}\right), 34.6\left(\mathrm{CH}_{2}\right), 53.0\left(\mathrm{CH}_{3}\right), 52.4$ $(\mathrm{CH}), 129.3\left(\mathrm{CH}_{\mathrm{Ar}}\right), 131.1\left(\mathrm{CH}_{\mathrm{Ar}}\right), 131.5\left(\mathrm{C}_{\mathrm{Ar}}\right), 134.2\left(\mathrm{C}_{\mathrm{Ar}}\right), 170.1(\mathrm{CO}), 171.1(\mathrm{CO}) ; \mathrm{IR}: v\left(\mathrm{~cm}^{-1}\right)$ 3269, 3072, 2953, 2914, 2856, 1757, 1733, 1652, 1549, 1430, 1376,1249, 1216, 1125, 1031, 841; MS (m/z, EI): 281 (M+25), 222 (45), 189 (8), 163 (100), 151 (42), 135 (19), 122 (14), 105 (51), 91 (36), 77 (34), 59 (35); Anal. Calcd for $\mathrm{C}_{14} \mathrm{H}_{19} \mathrm{NO}_{3} \mathrm{~S}$; C 59.76, H 6.81, N 4.98, O 17.06, S 11.40; Found: C 59.72, H 6.96, N 4.81, O 16.94, S 11.42.

2-Acetamido-3-(3,4-dimethylphenylsulfanyl)propionic acid methyl ester (3f). White solid, mp: 84$85^{\circ} \mathrm{C}$; ${ }^{1} \mathrm{H}-\mathrm{NMR}\left(\mathrm{CD}_{3} \mathrm{OD}\right): \delta(\mathrm{ppm}) 1.92\left(\mathrm{~s}, 3 \mathrm{H}, \mathrm{COCH}_{3}\right), 2.20\left(\mathrm{~s}, 3 \mathrm{H}, \mathrm{CH}_{3}-\mathrm{Ar}(4)\right), 2.21$ (s, $3 \mathrm{H}, \mathrm{CH}_{3}-$ $\operatorname{Ar}(3)), 3.14\left(\mathrm{dd},{ }^{2} J=14.0 \mathrm{~Hz},{ }^{3} J=8.0 \mathrm{~Hz}, 1 \mathrm{H}, \mathrm{SCH}_{2}\right), 3.31\left(\mathrm{dd},{ }^{2} J=14.0 \mathrm{~Hz},{ }^{3} J=4.8 \mathrm{~Hz}, 1 \mathrm{H}\right.$, $\left.\mathrm{SCH}_{2}\right), 3.61\left(\mathrm{~s}, 3 \mathrm{H}, \mathrm{OCH}_{3}\right), 4.53\left(\mathrm{dd},{ }^{3} \mathrm{~J}=8.0 \mathrm{~Hz},{ }^{3} \mathrm{~J}=4.8 \mathrm{~Hz}, 1 \mathrm{H}, \mathrm{CH}_{2}-\mathrm{CH}\right), 4.84(\mathrm{~s}, 1 \mathrm{H}, \mathrm{NH}), 7.05$ (m, $1 \mathrm{H}, \mathrm{Ar}-\mathrm{H}), 7.13(\mathrm{~m}, 1 \mathrm{H}, \mathrm{Ar}-\mathrm{H}), 7.19$ (m, $1 \mathrm{H}, \mathrm{Ar}-\mathrm{H}) ;{ }^{13} \mathrm{C}-\mathrm{NMR}\left(\mathrm{CD}_{3} \mathrm{OD}\right): \delta(\mathrm{ppm}) 19.5\left(\mathrm{CH}_{3}\right)$, $19.9\left(\mathrm{CH}_{3}\right), 22.4\left(\mathrm{CH}_{3}\right), 37.3\left(\mathrm{CH}_{2}\right), 52.9\left(\mathrm{CH}_{3}\right), 53.8\left(\mathrm{CH}_{3}\right), 130.2\left(\mathrm{CH}_{\mathrm{Ar}}\right), 131.4\left(\mathrm{CH}_{\mathrm{Ar}}\right), 132.6\left(\mathrm{C}_{\mathrm{Ar}}\right)$, $133.7\left(\mathrm{CH}_{\mathrm{Ar}}\right), 137.2\left(\mathrm{C}_{\mathrm{Ar}}\right), 138.7\left(\mathrm{C}_{\mathrm{Ar}}\right), 172.5(\mathrm{CO}), 173.2(\mathrm{CO})$; IR: $v\left(\mathrm{~cm}^{-1}\right)$ 3284, 3061, 3015, 2951, 2928, 2867, 1747, 1659, 1539, 1488, 1436, 1372, 1213, 1129, 1022, 814; MS (m/z, EI): $281\left(\mathrm{M}^{+}, 18\right)$, 222 (65), 191 (7), 163 (100), 151 (55), 137 (16), 105 (53), 91 (38), 77 (49), 59 (28); Anal. Calcd for $\mathrm{C}_{14} \mathrm{H}_{19} \mathrm{NO}_{3} \mathrm{~S}$; C 59.76, H 6.81, N 4.98, O 17.06, S 11.40; Found: C 59.87, H 6.98, N 4.93, O 16.84, S 11.19 .

2-Acetamido-3-(2,4-dimethylphenylsulfanyl)propionic acid methyl ester (3g). White solid, mp: 84$85^{\circ} \mathrm{C}$; ${ }^{1} \mathrm{H}-\mathrm{NMR}\left(\mathrm{CD}_{3} \mathrm{OD}\right): \delta(\mathrm{ppm}) 1.95\left(\mathrm{~s}, 3 \mathrm{H}, \mathrm{COCH}_{3}\right), 2.27\left(\mathrm{~s}, 3 \mathrm{H}, \mathrm{CH}_{3}-\mathrm{Ar}(4)\right), 2.36\left(\mathrm{~s}, 3 \mathrm{H}, \mathrm{CH}_{3}-\right.$ $\operatorname{Ar}(2)), 3.13\left(\mathrm{dd},{ }^{2} J=14.0 \mathrm{~Hz},{ }^{3} J=8.0 \mathrm{~Hz}, 1 \mathrm{H}, \mathrm{SCH}_{2}\right), 3.30\left(\mathrm{dd},{ }^{2} J=14.0 \mathrm{~Hz},{ }^{3} J=5.0 \mathrm{~Hz}, 1 \mathrm{H}\right.$, $\left.\mathrm{SCH}_{2}\right), 3.62\left(\mathrm{~s}, 3 \mathrm{H}, \mathrm{OCH}_{3}\right), 4.52\left(\mathrm{dd},{ }^{3} J=8.0 \mathrm{~Hz},{ }^{3} J=5.0 \mathrm{~Hz}, 1 \mathrm{H}, \mathrm{CH}_{2}-\mathrm{CH}\right), 4.86(\mathrm{~s}, 1 \mathrm{H}, \mathrm{NH}), 6.98$ 
(m, $1 \mathrm{H}, \mathrm{Ar}-\mathrm{H}), 7.04(\mathrm{~m}, 1 \mathrm{H}, \mathrm{Ar}-\mathrm{H}), 7.31(\mathrm{~m}, 1 \mathrm{H}, \mathrm{Ar}-\mathrm{H}) ;{ }^{13} \mathrm{C}-\mathrm{NMR}\left(\mathrm{CD}_{3} \mathrm{OD}\right): \delta(\mathrm{ppm}) 20.8\left(\mathrm{CH}_{3}\right)$, $21.2\left(\mathrm{CH}_{3}\right), 22.4\left(\mathrm{CH}_{3}\right), 36.5\left(\mathrm{CH}_{2}\right), 53.0\left(\mathrm{CH}_{3}\right), 53.7(\mathrm{CH}), 128.5\left(\mathrm{CH}_{\mathrm{Ar}}\right), 131.3\left(\mathrm{C}_{\mathrm{Ar}}\right), 132.4\left(\mathrm{CH}_{\mathrm{Ar}}\right)$, $132.9\left(\mathrm{CH}_{\mathrm{Ar}}\right), 138.6\left(\mathrm{C}_{\mathrm{Ar}}\right), 140.7\left(\mathrm{C}_{\mathrm{Ar}}\right), 172.5(\mathrm{CO}), 173.2(\mathrm{CO})$; IR: $v\left(\mathrm{~cm}^{-1}\right)$ 3293, 3063, 2959, 2916, 1749, 1652, 1546, 1435, 1411, 1376, 1292, 1258, 1218, 1170, 1127, 821; MS (m/z, EI): $281\left(\mathrm{M}^{+}, 48\right)$, 222 (91), 207 (11), 190 (10), 163 (70), 151 (63), 138 (29), 122 (18), 105 (100), 91 (52), 77 (74), 59 (54); Anal. Calcd for $\mathrm{C}_{14} \mathrm{H}_{19} \mathrm{NO}_{3} \mathrm{~S}$; C 59.76, H 6.81, N 4.98, O 17.06, S 11.40; Found: C 59.76, H 6.86, N 4.82, O 17.40, S 11.38.

2-Acetamido-3-(2,5-dimethylphenylsulfanyl)propionic acid methyl ester (3h). White solid, mp: 84$85^{\circ} \mathrm{C}$; ${ }^{1} \mathrm{H}-\mathrm{NMR}\left(\mathrm{CD}_{3} \mathrm{OD}\right): \delta(\mathrm{ppm}) 1.95\left(\mathrm{~s}, 3 \mathrm{H}, \mathrm{COCH}_{3}\right), 2.29\left(\mathrm{~s}, 3 \mathrm{H}, \mathrm{CH}_{3}-\mathrm{Ar}(5)\right), 2.33\left(\mathrm{~s}, 3 \mathrm{H}, \mathrm{CH}_{3}-\right.$ $\operatorname{Ar}(2)), 3.18\left(\mathrm{dd},{ }^{2} J=14.0 \mathrm{~Hz},{ }^{3} J=8.0 \mathrm{~Hz}, 1 \mathrm{H}, \mathrm{SCH}_{2}\right), 3.35\left(\mathrm{dd},{ }^{2} J=14,0 \mathrm{~Hz},{ }^{3} J=4.8 \mathrm{~Hz}, 1 \mathrm{H}\right.$, $\left.\mathrm{SCH}_{2}\right), 3.63\left(\mathrm{~s}, 3 \mathrm{H}, \mathrm{OCH}_{3}\right), 4.57\left(\mathrm{dd},{ }^{3} J=8.0 \mathrm{~Hz},{ }^{3} J=4.8 \mathrm{~Hz}, 1 \mathrm{H}, \mathrm{CH}_{2}-\mathrm{CH}\right), 4.86(\mathrm{~s}, 1 \mathrm{H}, \mathrm{NH}), 6.95$ $\left(\mathrm{d},{ }^{3} \mathrm{~J}=7.8 \mathrm{~Hz}, 1 \mathrm{H}, 1 \mathrm{Ar}-\mathrm{H}\right), 7.07\left(\mathrm{~d},{ }^{3} \mathrm{~J}=7.8 \mathrm{~Hz}, 1 \mathrm{H}, 1 \mathrm{Ar}-\mathrm{H}\right), 7.24$ (s, $\left.1 \mathrm{H}, 1 \mathrm{Ar}-\mathrm{H}\right) ;{ }^{13} \mathrm{C}-\mathrm{NMR}$ $\left(\mathrm{CD}_{3} \mathrm{OD}\right): \delta(\mathrm{ppm}) 20.3\left(\mathrm{CH}_{3}\right), 21.1\left(\mathrm{CH}_{3}\right), 22.4\left(\mathrm{CH}_{3}\right), 35.9\left(\mathrm{CH}_{2}\right), 53.0\left(\mathrm{CH}_{3}\right), 53.7(\mathrm{CH}), 129.0$ $\left(\mathrm{CH}_{\mathrm{Ar}}\right), 131.4\left(\mathrm{CH}_{\mathrm{Ar}}\right), 132.2\left(\mathrm{CH}_{\mathrm{Ar}}\right), 134.7\left(\mathrm{C}_{\mathrm{Ar}}\right), 137.0\left(\mathrm{C}_{\mathrm{Ar}}\right), 137.4\left(\mathrm{C}_{\mathrm{Ar}}\right), 172.4(\mathrm{CO}), 173.3(\mathrm{CO})$; IR: $v\left(\mathrm{~cm}^{-1}\right) 3319,3004,2952,2915,1769,1651,1530,1489,1432,1379,1251,1216,1189,1171,1029$, 976, 800; MS (m/z, EI): $281\left(\mathrm{M}^{+}, 25\right), 222$ (73), 190 (10), 163 (100), 149 (98), 135 (25), 122 (15), 105 (84), 91 (58), 77 (78), 59 (58); Anal. Calcd for $\mathrm{C}_{14} \mathrm{H}_{19} \mathrm{NO}_{3} \mathrm{~S}$; C 59.76, H 6.81, N 4.98, O 17.06, S 11.40; Found: C 59.91, H 6.84, N 4.95, O 17.41, S 11.52.

2-Acetamido-3-phenylsulfanylpropionic acid methyl ester (3i). White solid, mp: $64-65^{\circ} \mathrm{C}$; ${ }^{1} \mathrm{H}-\mathrm{NMR}$ $\left(\mathrm{CD}_{3} \mathrm{OD}\right): \delta(\mathrm{ppm}) 1.96$ (s, $\left.3 \mathrm{H}, \mathrm{COCH}_{3}\right), 3.23\left(\mathrm{dd},{ }^{2} J=14.0 \mathrm{~Hz},{ }^{3} J=8.0 \mathrm{~Hz}, 1 \mathrm{H}, \mathrm{SCH}_{2}\right), 3.40\left(\mathrm{dd},{ }^{2} J\right.$ $\left.=14,0 \mathrm{~Hz},{ }^{3} J=5.2 \mathrm{~Hz}, 1 \mathrm{H}, \mathrm{SCH}_{2}\right), 3.62\left(\mathrm{~s}, 3 \mathrm{H}, \mathrm{OCH}_{3}\right), 4.68\left(\mathrm{dd},{ }^{3} J=8.0 \mathrm{~Hz},{ }^{3} J=5.2 \mathrm{~Hz}, 1 \mathrm{H}, \mathrm{CH}_{2}-\right.$ $\mathrm{CH}), 4.85$ (s, 1H, NH), 7.20-7.24 (m, 1 H, 1 Ar-H), 7.28-7.32 (m, 2 H, 2 Ar-H), 7.39-7.43 (m, 2 H, 2 Ar-H); ${ }^{13} \mathrm{C}-\mathrm{NMR}\left(\mathrm{CD}_{3} \mathrm{OD}\right): \delta(\mathrm{ppm}) 22.4\left(\mathrm{CH}_{3}\right), 36.6\left(\mathrm{CH}_{2}\right), 53.0\left(\mathrm{CH}_{3}\right), 53.8(\mathrm{CH}), 128.1\left(\mathrm{CH}_{\mathrm{Ar}}\right)$, $130.3\left(\mathrm{CH}_{\mathrm{Ar}}\right), 131.8\left(\mathrm{CH}_{\mathrm{Ar}}\right), 136.3\left(\mathrm{C}_{\mathrm{Ar}}\right), 172.4(\mathrm{CO}), 173.3(\mathrm{CO})$; IR: $v\left(\mathrm{~cm}^{-1}\right)$ 3283, 3058, 2952, 2849, 1747, 1653, 1539, 1482, 1438, 1373, 1306, 1214, 1177, 1128, 1025, 743; MS (m/z, EI): 253 $\left(\mathrm{M}^{+}, 10\right), 194$ (84), 163 (14), 135 (100), 123 (73), 109 (45), 88 (42), 77 (35), 65 (47); Anal. Calcd for $\mathrm{C}_{12} \mathrm{H}_{15} \mathrm{NO}_{3} \mathrm{~S}$; C 56.90, H 5.97, N 5.53, O 18.95, S 12.66; Found: C, 57.35; H, 5.91; N, 5.32; O, 18.75; $\mathrm{S}, 12.66$.

2-Acetamido-3-(4-chlorophenylsulfanyl)propionic acid methyl ester (3j). White solid, mp: 106-107 ${ }^{\circ}$; ${ }^{1} \mathrm{H}-\mathrm{NMR}\left(\mathrm{DMSO}_{6}\right): \delta(\mathrm{ppm}) 1.83\left(\mathrm{~s}, 3 \mathrm{H}, \mathrm{COCH}_{3}\right), 3.19\left(\mathrm{dd},{ }^{2} J=13.8 \mathrm{~Hz},{ }^{3} J=8.0 \mathrm{~Hz}, 1 \mathrm{H}, \mathrm{SCH}_{2}\right)$, $3.32\left(\mathrm{dd},{ }^{2} J=13.8 \mathrm{~Hz},{ }^{3} J=5.4 \mathrm{~Hz}, 1 \mathrm{H}, \mathrm{SCH}_{2}\right), 3.57\left(\mathrm{~s}, 3 \mathrm{H}, \mathrm{OCH}_{3}\right), 4.40\left(\mathrm{dd},{ }^{3} J=8.0 \mathrm{~Hz},{ }^{3} J=8.0\right.$ $\left.\mathrm{Hz},{ }^{3} J=5.4 \mathrm{~Hz}, 1 \mathrm{H}, \mathrm{CH}-\mathrm{CH}_{2}\right), 7.36$ (s, $\left.4 \mathrm{H}, \mathrm{Ar}-\mathrm{H}\right), 8.50\left(\mathrm{~d},{ }^{3} J=8.0 \mathrm{~Hz}, 1 \mathrm{H}, \mathrm{NH}\right) ;{ }^{13} \mathrm{C}-\mathrm{NMR}$ (DMSO$\left.\mathrm{d}_{6}\right): \delta(\mathrm{ppm}) 22.4\left(\mathrm{CH}_{3}\right), 34.6\left(\mathrm{CH}_{2}\right), 51.8\left(\mathrm{CH}_{3}\right), 52.4(\mathrm{CH}), 129.3\left(\mathrm{CH}_{\mathrm{Ar}}\right), 131.1\left(\mathrm{CH}_{\mathrm{Ar}}\right), 131.5\left(\mathrm{C}_{\mathrm{Ar}}\right)$, $134.2\left(\mathrm{C}_{\mathrm{Ar}}\right), 170.1(\mathrm{CO}), 171.1(\mathrm{CO})$; IR: $v\left(\mathrm{~cm}^{-1}\right)$ 3308, 3075, 2951, 2930, 1730, 1643, 1546, 1478, 1425, 1369, 1349, 1247, 1219, 1192, 1177, 1099, 1037, 1011, 808; MS (m/z, EI): $287\left(\mathrm{M}^{+}, 11\right), 230$ (41), 228 (100), 197 (10), 169 (50), 157 (22), 143 (15), 108 (12), 88 (29); Anal. Calcd for $\mathrm{C}_{12} \mathrm{H}_{14} \mathrm{ClNO}_{3} \mathrm{~S}$; C 50.09, H 4.90, $\mathrm{Cl} 12.32, \mathrm{~N} 4.87$, O 16.68, S 11.14; Found: C 50.80, H 5.23, $\mathrm{Cl}$ 11.93, N 4.68, O 16.20, S 10.54. 
2-Acetamido-3-(4-bromophenylsulfanyl)propionic acid methyl ester (3k). White solid, mp: 121-122 ${ }^{\circ} \mathrm{C}$; ${ }^{1} \mathrm{H}-\mathrm{NMR}\left(\mathrm{CDCl}_{3}\right): \delta(\mathrm{ppm}) 1.80\left(\mathrm{~s}, 3 \mathrm{H}, \mathrm{COCH}_{3}\right), 3.21\left(\mathrm{dd},{ }^{2} J=14.2 \mathrm{~Hz},{ }^{3} J=4.7 \mathrm{~Hz}, 1 \mathrm{H}, \mathrm{SCH}_{2}\right), 3.35$ $\left(\mathrm{dd},{ }^{2} J=14.2 \mathrm{~Hz},{ }^{3} J=4.7 \mathrm{~Hz}, 1 \mathrm{H}, \mathrm{SCH}_{2}\right), 3.49\left(\mathrm{~s}, 3 \mathrm{H}, \mathrm{OCH}_{3}\right), 4.73\left(\mathrm{~m}, 1 \mathrm{H}, \mathrm{CH}-\mathrm{CH}_{2}\right), 6.16(\mathrm{~s}, 1 \mathrm{H}$, $\mathrm{NH}), 7,14-7,16(\mathrm{~m}, 2 \mathrm{H}, \mathrm{Ar}-\mathrm{H}), 7.29-7.31$ (m, $2 \mathrm{H}, \mathrm{Ar}-\mathrm{H}) ;{ }^{13} \mathrm{C}-\mathrm{NMR}\left(\mathrm{CDCl}_{3}\right)$ : $\delta$ (ppm) $22.9\left(\mathrm{CH}_{3}\right)$, $36.5\left(\mathrm{CH}_{2}\right), 52.2\left(\mathrm{OCH}_{3}\right), 52.5(\mathrm{CH}), 121.0\left(\mathrm{C}_{\mathrm{Ar}}\right), 132.1\left(\mathrm{CH}_{\mathrm{Ar}}\right), 132.3\left(\mathrm{CH}_{\mathrm{Ar}}\right), 134.0\left(\mathrm{C}_{\mathrm{Ar}}\right), 169.6$ (CO), 170.6 (CO); IR: $v\left(\mathrm{~cm}^{-1}\right)$ 3307, 3047, 2950, 1729, 1641, 1546, 1474, 1425, 1350, 1324, 1245, 1176, 1092, 1039, 1006, 806; MS (m/z, EI): 333 (18), $331\left(\mathrm{M}^{+}, 17\right), 274$ (99), 272 (100), 215 (10), 213 (20), 203 (13), 201 (13), 189 (11), 187 (11), 144 (18), 134 (53), 122 (67), 108 (50), 88 (74), 75 (25), 60 (60); Anal. Calcd for $\mathrm{C}_{12} \mathrm{H}_{14} \mathrm{BrNO}_{3} \mathrm{~S}$ : C, 43.38; H, 4.25; Br, 24.05; N, 4.22; O, 14.45; S, 9.65. Found: C,44.56, H, 4.41, Br, 23.59, N, 4.04, O, 14.38, S, 9.29.

2-Acetamido-3-(4-methoxyphenylsulfanyl)propionic acid methyl ester (3I). Colorless oil; ${ }^{1} \mathrm{H}-\mathrm{NMR}$ $\left(\mathrm{CDCl}_{3}\right): \delta(\mathrm{ppm}) 1.89\left(\mathrm{~s}, 3 \mathrm{H}, \mathrm{COCH}_{3}\right), 3.22\left(\mathrm{dd},{ }^{2} J=14.2 \mathrm{~Hz},{ }^{3} J=5.0 \mathrm{~Hz}, 1 \mathrm{H}, \mathrm{SCH}_{2}\right), 3.32\left(\mathrm{dd},{ }^{2} J=\right.$ $\left.14.2 \mathrm{~Hz},{ }^{3} J=4.7 \mathrm{~Hz}, 1 \mathrm{H}, \mathrm{SCH}_{2}\right), 3.54\left(\mathrm{~s}, 3 \mathrm{H}, \mathrm{OCH}_{3}\right), 3.76\left(\mathrm{~s}, 3 \mathrm{H}, \mathrm{Ar}-\mathrm{OCH}_{3}\right), 4.77(\mathrm{~m}, 1 \mathrm{H}, \mathrm{CH}-$ $\left.\mathrm{CH}_{2}\right), 6.81-6.83(\mathrm{~m}, 2 \mathrm{H}, \mathrm{Ar}-\mathrm{H}), 7.35-7.37$ (m, $\left.2 \mathrm{H}, \mathrm{Ar}-\mathrm{H}\right) ;{ }^{13} \mathrm{C}-\mathrm{NMR}\left(\mathrm{CDCl}_{3}\right)$ : $\delta(\mathrm{ppm}) 22.9\left(\mathrm{CH}_{3}\right)$, $38.0\left(\mathrm{CH}_{2}\right), 52.2\left(\mathrm{OCH}_{3}\right), 52.3(\mathrm{CH}), 55.3\left(\mathrm{OCH}_{3}\right), 114.7\left(\mathrm{CH}_{\mathrm{Ar}}\right), 124.7\left(\mathrm{C}_{\mathrm{Ar}}\right), 134.2\left(\mathrm{CH}_{\mathrm{Ar}}\right), 159.4$ $\left(\mathrm{C}_{\mathrm{Ar}}\right), 169.7(\mathrm{CO}), 170.8(\mathrm{CO})$; IR: $v\left(\mathrm{~cm}^{-1}\right)$ 3289, 3065, 2953, 2838, 1746, 1656, 1592, 1541, 1494, 1437, 1373, 1286, 1246, 1175, 1029, 827; MS (m/z, EI): 283 (47), 224 (100), 165 (27), 153 (22), 144 (25), 165 (27), 109 (9), 84 (7); Anal. Calcd for $\mathrm{C}_{13} \mathrm{H}_{17} \mathrm{NO}_{4} \mathrm{~S}: \mathrm{C}, 55.11$; H, 6.05; N, 4.94; O, 22.59; S, 11.32. Found: C, 55.27; H, 6.32; N, 4.87; O, 22.79; S, 11.30 .

2-Acetamido-3-pentachlorophenylsulfanylpropionic acid methyl ester (3m). White solid, mp: 145 $146^{\circ} \mathrm{C}$; ${ }^{1} \mathrm{H}-\mathrm{NMR}\left(\mathrm{DMSO}_{\mathrm{d}}\right.$ ): $\delta(\mathrm{ppm}) 11.75\left(\mathrm{~s}, 3 \mathrm{H}, \mathrm{COCH}_{3}\right), 3.17\left(\mathrm{dd},{ }^{2} J=14.0 \mathrm{~Hz},{ }^{3} \mathrm{~J}=8.8 \mathrm{~Hz}, 1\right.$ $\left.\mathrm{H}, \mathrm{SCH}_{2}\right), 3.40\left(\mathrm{dd},{ }^{2} J=14.0 \mathrm{~Hz},{ }^{3} J=4.8 \mathrm{~Hz}, 1 \mathrm{H}, \mathrm{SCH}_{2}\right), 3.57\left(\mathrm{~s}, 3 \mathrm{H}, \mathrm{OCH}_{3}\right), 4.36\left(\mathrm{ddd},{ }^{3} J=8.8 \mathrm{~Hz}\right.$, $\left.{ }^{3} J=8.0 \mathrm{~Hz},{ }^{3} J=4.8 \mathrm{~Hz}, 1 \mathrm{H}, \mathrm{CH}-\mathrm{CH}_{2}\right), 8.36\left(\mathrm{~d},{ }^{3} \mathrm{~J}=8.0 \mathrm{~Hz}, 1 \mathrm{H}, \mathrm{NH}\right) ;{ }^{13} \mathrm{C}-\mathrm{NMR}\left(\mathrm{DMSO}-\mathrm{d}_{6}\right): \delta(\mathrm{ppm})$ $22.4\left(\mathrm{CH}_{3}\right), 36.0\left(\mathrm{CH}_{2}\right), 52.5(\mathrm{CH}), 52.8\left(\mathrm{CH}_{3}\right), 131.4\left(\mathrm{C}_{\mathrm{Ar}}\right), 133.5\left(\mathrm{C}_{\mathrm{Ar}}\right), 134.4\left(\mathrm{C}_{\mathrm{Ar}}\right), 138.2\left(\mathrm{C}_{\mathrm{Ar}}\right)$, 170.0 (CO), 170.8 (CO); IR: $v\left(\mathrm{~cm}^{-1}\right)$ 3293, 2956, 1758, 1651, 1532, 1435, 1378, 1334, 1305, 1259, 1215, 1190, 1170, 1130, 688; MS (m/z, EI): 425 (M+ , 5), 366 (100), 324 (13), 246 (18), 167 (12), 144 (37), 117 (48), 102 (18), 88 (90), 69 (34); Anal. Calcd for $\mathrm{C}_{12} \mathrm{H}_{10} \mathrm{Cl}_{5} \mathrm{NO}_{3} \mathrm{~S}$; C 33.87, H 2.37, $\mathrm{Cl} 41.66$, N 3.29, O 11.28, S 7.54; Found: C 34.24, H 2.58, Cl 42.01, N 2.97, O 10.75, S 6.96.

\section{Acknowledgements}

The authors gratefully acknowledge Caroline Bastien and Stéphane Grossmann for their technical assistance.

\section{References and Notes}

1. Lauwerys, R.R.; Haufroid, V.; Hoet, P.; Lison, D. In Toxicologie industrielle et intoxications professionnelles; Elsevier Masson S.A.S. Ed.: Issy-les-Moulineaux (France), 2007; pp. 528-557.

2. Lataye, R.; Campo, P.; Pouyatos, B.; Cossec, B.; Blachere, V.; Morel, G. Solvent ototoxicity in the rat and guinea pig. Neurotox. Teratol. 2003, 25, 39-50. 
3. Gagnaire, F.; Marignac, B.; Langlais, C.; Bonnet, P. Ototoxicity in rats exposed to ortho-, metaand para-xylene vapours for 13 weeks. Pharmacol. Toxicol. 2001, 89, 6-14.

4. Korsak, Z.; Rydzynski, K. Neurotoxic effects of acute and subchronic inhalation exposure to trimethylbenzene isomers (pseudocumene, mesitylene, hemimellitene) in rats. Int. J. Occup. Med. Environ. Health. 1996, 9, 341-349.

5. Vermeulen, N.P.E. Analysis of mercapturic acids as a tool in biotransformation, biomonitoring and toxicological studies. Trends Pharmacol. Sci. 1989, 10, 177-181.

6. Nelson, E.D. Determination of mercapturic acid and excretions in exposure control to toxicants. Crit. Rev. Toxicol. 1992, 22, 371-389.

7. De Rooij, B.M.; Commandeur, J.N.M.; Vermeulen, N.P.E. Mercapturic acids as biomarkers of exposure to electrophilic chemicals: applications to environmental and industrial chemicals. Biomarkers 1998, 3, 239-303.

8. Angerer, J.; Ewers, U.; Wilhelm, M. Human biomonitoring: State of the art. Int. J. Hyg. Environ. Health 2007, 210, 201-228.

9. Perbellini, L.; Veronese, N.; Princivalle, A. Mercapturic acids in the biological monitoring of occupational exposure to chemicals. J. Chrom. B Analyt. Technol. Biomed. Life Sci. 2002, 781, 269-290.

10. Haufroid, V.; Lison, D. Mercapturic acids revisited as biomarkers of exposure to reactive chemicals in occupational toxicology: a minireview. Int. Arch. Occup. Environ. Health 2005, 78, 343-354.

11. Van Doorn, R.; Leijdekkers, C.M.; Bos, R.P.; Brouns, R.M.E.; Henderson, P.T. Alcohol and sulphate intermediates in the metabolism of toluene and xylenes to mercapturic acids. J. Appl. Toxicol. 1981, 1, 236-242.

12. Zheng, J.; Hanzlik, R.P. Bromo(monohydroxy)phenyl mercapturic acids from bromobenzenetreated rats. Drug Metabol. Disp. 1992, 20, 688-694.

13. Angerer, J.; Schildbach, M.; Kramer, A. S-p-Toluylmercapturic acid in the urine of workers exposed to toluene: a new biomarker for toluene exposure. Arch. Toxicol. 1998, 72, 119-123.

14. Gonzalez-Reche, L.M.; Schettgen, T.; Angerer, J. New approaches to the metabolism of xylenes: verification of the formation of phenylmercapturic acid metabolites of xylenes. Arch. Toxicol. 2003, 77, 80-85.

15. Boogaard, P.J.; Van Sittert, N.J. Biological monitoring of exposure to benzene - A comparison between S-phenylmercapturic acid, trans,trans-muconic acid and phenol. Occup. Environ. Med. 1995, 52, 611-620.

16. Inoue, O.; Kanno, E.; Kasai, K.; Ukai, H.; Okamoto, S.; Ikeda, M. Benzylmercapturic acid is superior to hippuric acid and o-cresol as a urinary marker of occupational exposure to toluene. Toxicol. Lett. 2004, 147, 177-186.

17. American Conference of Governmental Industrial Hygienists, Threshold Limit Values and Biological Exposure Indices 1998.

18. Deutsche Forschungsgemeinschaft, List of MAK and BAT values. Commission for the investigation of health hazards of chemical compounds in the work area, Report $N^{\circ} 32$. VCH: New-York, 1998. 
19. Van Bladeren, P.J.; Buys, W.; Breimer, D.D.; Van der Gen, A. The Synthesis of Mercapturic Acids and their Esters. Eur. J. Med. Chem. 1980, 15, 495-497.

20. Hayden, P.; Schaeffer, V.H.; Larsen, G.; Stevens, J.L. Cysteine S-conjugates. Meth. Enzymol. 1987, 143, 228-234.

21. Buijs, W.; Eid, M.I.A.; Onkenhout, W.; Vermeulen, N.P.E. The use of sulfenyl halides in the synthesis of mercapturic acids and their esters. Recl. Trav. Chim. Pays-Bas 1986, 105, 449-455.

22. West, H.D.; Mathura, G.R. Synthesis of some aryl-substituted L-cysteines and their fate in the animal body. J. Biol. Chem. 1954, 208, 315-318.

23. Parke, D.V.; Williams, R.T. Studies in detoxication 38. The metabolism of benzene: the determination of phenylmercapturic acid in urine; mercapturic acid excretion by rabbits receiving benzene. Biochem. J. 1951, 48, 624-629.

24. Ciattini, P.G.; Morera, E.; Ortar, G. A new, palladium-catalyzed synthesis of aromatic mercapturic acid derivatives. Tetrahedron Lett. 1995, 36, 4133-4136.

25. Hickman, R.J.S.; Christie, B.J.; Guy, R.W.; White, T.J. Synthesis of aromatic S-substituted derivatives of N-acetyl-L-cysteine. Aust. J. Chem. 1985, 38, 899-904.

26. Kondoh, A.; Yorimitsu, H.; Oshima, K. Nucleophilic aromatic substitution reaction of nitroarenes with alkyl- or arylthio groups in dimethyl sulfoxide by means of cesium carbonate . Tetrahedron 2006, 62, 2357-2360.

27. Pombrio, J.M.; Giangreco, A.; Li, L.Q.; Wempe, M.F.; Anders, M.W.; Swett, D.H.; Pritchard, J.B.; Ballatori, N. Mercapturic acids (N-acetylcysteine S-conjugates) as endogenous substrates for the renal organic anion transporter-1. Mol. Pharmacol. 2001, 60, 1091-1099.

28. Saxena, M.; Henderson, G.B. MOAT4, a novel multispecific organic-anion transporter for glucuronides and mercapturates in mouse L1210 cells and human erythrocytes. Biochem. J. 1996, 320, 273-281.

29. Nakamura, S.; Goto, K.; Kondo, M.; Naito, S.; Tsuda, Y.; Shishido, K. Enantioselective total synthesis and structure determination of the mercapturic acid sulfoxide conjugate. Bioorg. Med. Chem. Lett. 1997, 7, 2033-2036.

30. Behringer, H.; Fackler, E. Eine einfache synthese der racemischen Mercaptursäuren. Justus Liebigs Ann. Chem. 1949, 564, 73-78.

31. Hanzlik, R.P.; Weller, P.E.; Desai, J.; Zheng, J.; Hall, L.R; Slaughter, D.E. Synthesis of mercapturic acid-derivatives of putative toxic metabolites of bromobenzene. J. Org. Chem. 1990, 55, 2736-2742.

32. Enders, D.; Lüttgen, K.; Narine, A.A. Asymmetric sulfa-Michael additions. Synthesis 2007, 7, 959-980.

33. Montanari, F.; Landini, D.; Rolla, F. Phase transfer catalyzed reactions. Top. Curr. Chem. 1982, 101, 147-200.

34. Rothstein, E. $J$. Experiments in the synthesis of derivatives of $\alpha, \alpha$-aminoacrylic acid from serine and N-substituted serines. Chem. Soc. 1949, 1968-1971.

35. Cardillo, G.; Gentilucci, L.; Tolomelli, A.; Tomasini, C. Asymmetric 1,4 addition of Grignard reagents to chiral alpha,beta-unsaturated esters in the presence of Lewis acids. Tetrahedron 1999, 55, 6231-6242. 
36. Bueno, M.P.; Cativiela, C.; Finol, C.; Mayoral, J.A.; Jaime, C. Diels-Alder reactions of methylN-acyl-alpha,beta-dehydroalaninates with cyclopentadiene. Can. J. Chem. 1987, 65, 2182-2186.

37. Roper, R.; Ma, T.S. Diazomethane as a reagent for microsynthesis. Microchemical J. 1957, 1 , 245-260.

38. Kolar, A.J.; Olsen, R.K. Convenient, large-scale preparation of 2-acetamidoacrylic acid and its methyl ester. Synthesis 1977, 457-459.

39. Rich, D.H.; Tam, J.P. Synthesis of didehydropeptides from peptides containing 3-alkylthioamino acid residues. Tetrahedron Lett. 1975, 211-212.

40. Srivastava, V.P.; Roberts, M.; Holmes, T.; Stammer, C.H. Synthesis of (+/-) 2,3-methanovaline and (+/-)-2,3-methanoleucine. J. Org. Chem. 1989, 54, 5866-5870.

41. Placidi-Rampont, V. Synthèse, séparation et analyse de métabolites soufrés du toluène. $\mathrm{PhD}$ Thesis, Henri Poincaré University Nancy 1: Nancy, France, 1997

42. Bastien, C. Synthèse et identification de métabolites soufrés du toluène. DEA report, Henri Poincaré University Nancy 1: Nancy, France, 2000

43. Wang, S.S.; Gisin, B.F.; Winter, D.P.; Makofske, R.; Kulesha, I.D.; Tzougraki, C.; Meienhofer, J. Facile synthesis of amino-acid and peptide esters under mild conditions via cesium salts. $J$. Org. Chem. 1977,42, 1286-1290.

44. Chidambaran, M.; Sonavane, S.U.; de la Zerda, J.; Sasson, Y. Didecyldimethylammonium bromide (DDAB): a universal, robust, and highly potent phase-transfer catalyst for diverse organic transformations. Tetrahedron 2007, 63, 7696-7701.

45. Makosza, M. Phase-transfer catalysis. A general green methodology in organic synthesis. Pure Appl. Chem. 2000, 72, 1399-1403.

46. Sirovski, F.; Reichardt, C.; Garokhova, M.; Ruban, S.; Stoikova, E. Solid liquid phase-transfer catalysis. Some models and solvent influence. Tetrahedron 1999, 55, 6363-6374.

47. Yang, H.M.; Lin, C.L. Phase-transfer catalyzed benzylation of sodium benzoate using aliquat 336 as catalyst in liquid-liquid system. J. Mol. Catal. A: Chem. 2003, 206, 67-76.

48. Lopez, A.; Moreno-Manas, M.; Pleixats, R.; Roglans, A.; Ezquerra, J.; Pedregal, C. Ethyl N(diphenylmethylene)glycinate as anionic glycine equivalent. Monoalkylation, dialkylation and Michael additions under solid-liquid phase-transfer catalysis. Tetrahedron 1996, 52, 8385-8386.

49. Herriott, A.W.; Picker, D. Phase transfer catalysis - evaluation of catalysts. J. Am. Chem. Soc. 1975, 97, 2345-2349.

50. Superchi, S.; Nardiello, M.; Donnoli, M.I.; Scafato, P.; Menicagli, R.; Rosini, C. Enantioselective synthesis of the fragrance trans-magnolione under asymmetric phase transfer catalysis. C.R. Chimie 2005, 8, 867-874.

Sample Availability: Samples of the compounds mentioned in this paper are available from the corresponding author (B.C.)

(C) 2008 by the authors; licensee Molecular Diversity Preservation International, Basel, Switzerland. This article is an open-access article distributed under the terms and conditions of the Creative Commons Attribution license (http://creativecommons.org/licenses/by/3.0/). 